\title{
Loss-of-Function Mutations in Human Regulator of G Protein Signaling RGS2 Differentially Regulate Pharmacological Reactivity of Resistance Vasculature ${ }^{[\mathbf{S}}$
}

\author{
(1) Hoa T.N. Phan, ${ }^{1}$ William F. Jackson, Vincent S. Shaw, Stephanie W. Watts, \\ and Richard R. Neubig \\ Department of Pharmacology and Toxicology, Michigan State University, East Lansing, Michigan (H.T.N.P., W.F.J., V.S.S., \\ S.W.W., R.R.N.)
}

Received April 4, 2019; accepted October 3, 2019

\begin{abstract}
Regulator of G protein signaling 2 (RGS2) plays a role in reducing vascular contraction and promoting relaxation due to its GTPase accelerating protein activity toward $\mathrm{G} \alpha \mathrm{q}$. Previously, we identified four human loss-of-function (LOF) mutations in RGS2 (Q2L, $\mathrm{D} 40 \mathrm{Y}, \mathrm{R} 44 \mathrm{H}$, and $\mathrm{R} 188 \mathrm{H})$. This study aimed to investigate whether those RGS2 LOF mutations disrupt the ability of RGS2 to regulate vascular reactivity. Isolated mesenteric arteries (MAs) from RGS2 ${ }^{-/-}$mice showed an elevated contractile response to $5 \mathrm{nM}$ angiotensin II and a loss of acetylcholine (ACh)-mediated vasodilation. Reintroduction of a wild-type (WT) RGS2-GFP plasmid into RGS2 ${ }^{-1-}$ MAs suppressed the vasoconstrictor response to angiotensin II. RGS2 LOF mutants failed to suppress the angiotensin II constriction response compared with RGS2 WT. In contrast, ACh-mediated vasoconstriction was restored by expression of RGS2 WT, D40Y, and R44H but not by RGS2 Q2L or R188H. Phosphorylation of RGS2 D40Y and R44H by protein kinase $\mathrm{G}$ (PKG) may explain their maintained function to support relaxation in MAs. This is supported by phosphomimetic mutants and suppression of vasorelaxation mediated by RGS2 D40Y by a PKG inhibitor. These results demonstrate that RGS2 attenuates vasoconstriction in MAs and that RGS2 LOF mutations cannot carry out this effect. Among them, the Q2L and
\end{abstract}

$\mathrm{R} 188 \mathrm{H}$ mutants supported less relaxation to $\mathrm{ACh}$, whereas relaxation mediated by the $\mathrm{D} 40 \mathrm{Y}$ and $\mathrm{R} 44 \mathrm{H}$ mutant proteins was equal to that with WT protein. Phosphorylation of RGS2 by PKG appears to contribute to this vasorelaxation. These results provide insights for precision medicine targeting the rare individuals carrying these RGS2 mutations.

\section{SIGNIFICANCE STATEMENT}

Regulator of G protein signaling 2 (RGS2) has been implicated in the control of blood pressure; rare mutations in the RGS2 gene have been identified in large-scale human gene sequencing studies. Four human mutations in RGS2 that cause loss of function (LOF) in cell-based assays were examined in isolated mouse arteries for effects on both vasoconstriction and vasodilation. All mutants showed the expected LOF effects in suppressing vasoconstriction. Surprisingly, the $\mathrm{D} 40 \mathrm{Y}$ and $\mathrm{R} 44 \mathrm{H}$ mutant RGS2 showed normal control of vasodilation. We propose that this is due to rescue of the mislocalization phenotype of these two mutants by nitric oxide-mediated/protein kinase $\mathrm{G}-$ dependent phosphorylation. These mechanisms may guide drug discovery or drug repurposing efforts for hypertension by enhancing RGS2 function.

\section{Introduction}

$\mathrm{G}$ protein-mediated signaling is critical for the regulation of cardiovascular function, including heart rate, cell growth, and vascular tone. Blockers of $\mathrm{G}$ protein-coupled receptor signaling (angiotensin receptor and $\beta$ and $\alpha$ adrenergic receptor blockers) are among the best therapies for hypertension and

This work was supported by Michigan State University and a predoctoral fellowship from the American Heart Association for HTN Phan.

${ }^{1}$ Current affiliation: Department of Pharmacology, University of Michigan, Ann Arbor, Michigan.

https://doi.org/10.1124/mol.119.116822.

S This article has supplemental material available at molpharm. aspetjournals.org. heart failure. Regulator of G protein signaling (RGS) proteins accelerate the rate of GTP hydrolysis by active $\mathrm{G} \alpha \mathrm{i} / \mathrm{o}$ and $\mathrm{G} \alpha \mathrm{q}$ subunits, thereby reducing the amplitude and duration of $\mathrm{G}$ protein-coupled receptor/G protein signaling. Among more than 20 RGS proteins, RGS2 is highly expressed in vascular smooth muscle cells (VSMCs), where it is regulated by nitric oxide (NO) and controls vascular constriction and relaxation (Tang et al., 2003; Sun et al., 2005).

Biochemically, RGS2 negatively regulates G $\alpha$, which transduces signals from a variety of vasoconstrictors. Consistent with a role in vascular control, RGS2 knockout mice are hypertensive (Heximer et al., 2003) and hypertensive human patients had reduced RGS2 mRNA in peripheral

ABBREVIATIONS: ACh, acetylcholine; Angll, angiotensin II; $\mathrm{CHO}$, Chinese hamster ovary; EDHF, endothelium-derived hyperpolarizing factor; GAP, GTPase accelerating protein; HEK, human embryonic kidney; LOF, loss of function; MA, mesenteric artery; NO, nitric oxide; PB, phosphate buffer; PE, phenylephrine; PKG, protein kinase G; PM, plasma membrane; PSS, physiologic salt solution; RGS, regulator of G protein signaling; SNP, single nucleotide polymorphism; TES, 2-\{[2-hydroxy-1,1-bis(hydroxymethyl)ethyl]amino\}ethanesulfonic acid; VSMC, vascular smooth muscle cell; WT, wild type. 
blood mononuclear cells compared with controls (Semplicini et al., 2006). Furthermore, hypertensive patients were more likely to have the single nucleotide polymorphism (SNP) C1114G in the $3^{\prime}$ untranslated region of the RGS2 gene, which correlated with lower RGS2 protein expression in cultured fibroblasts isolated from skin biopsy (Semplicini et al., 2006). Several rare coding SNPs were found in a Japanese hypertensive cohort at a frequency higher than normotensive controls but the sample size was too small to reach statistical significance (Yang et al., 2005). Subsequent studies confirmed loss of function (LOF) of these SNPs in vitro and defined mechanisms (Bodenstein et al., 2007; $\mathrm{Gu}$ et al., 2008). We recently identified four LOF mutations in human RGS2 (Phan et al., 2017) from a set of 16 variants of unknown significance found in the Genome Aggregation Database (Lek et al., 2016). Although they are biochemically characterized, the functional consequences of these mutations in the complex milieu of vascular tissue are unknown. Here, we used an ex vivo method in mesenteric arteries (MAs) to examine how these mutations affect vascular reactivity. We hypothesized that the LOF phenotype of RGS2 mutants identified in a cell-based assay would have LOF physiologic consequence in the MAs.

\section{Materials and Methods}

Ethical Approval. All experimental procedures were approved by the Michigan State University Animal Care and Use Committee and were performed in accordance with the Guide for the Care and Use of Laboratory Animals established by the National Institutes of Health.

Animals. C57Bl/6 mice with wild type (WT) and RGS2 $2^{-1-}$ genotypes were obtained from Dr. Kendall Blumer (Washington University St. Louis) (Osei-Owusu et al., 2015). Mice were provided access to food and water ad libitum in the Michigan State University animal facility on a 12-hour light/dark cycle. All of the experiments were performed using 3- to 5-month-old male mice. Mice were anesthetized by isoflurane, the MA was removed, and they were then euthanized by cervical dislocation.

Materials. Rp-8-pCPT-cGMPS (item 18445, CAS 208445-07-2) was purchased from Cayman Chemical. Angiotensin II (AngII; A9525), phenylephrine (PE; P6126), and acetylcholine (ACh; A6625) were purchased from Sigma-Aldrich (St. Louis, MO).

Pressure Myography. MAs were isolated free of adipose and connective tissue in a physiologic salt solution (PSS) containing $140 \mathrm{mM} \mathrm{NaCl}, 5 \mathrm{mM} \mathrm{KCl}, 1 \mathrm{mM} \mathrm{MgCl}{ }_{2} \cdot 6 \mathrm{H}_{2} \mathrm{O}, 10 \mathrm{mM}$ HEPES, and $10 \mathrm{mM}$ dextrose, $\mathrm{pH} \mathrm{7.4}$, at $4^{\circ} \mathrm{C}$. MAs were mounted between two glass micropipettes in a custom-made cannulation chamber and were visualized on the stage of an inverted microscope (Zeiss Axiovert 35) as previously described (Diaz-Otero et al., 2016). Arteries were pressurized and then equilibrated for 60 minutes in PSS containing $140 \mathrm{mM} \mathrm{NaCl}, 5 \mathrm{mM} \mathrm{KCl}, 1.8 \mathrm{mM} \mathrm{CaCl} 2 \cdot 2 \mathrm{H}_{2} \mathrm{O}, 1 \mathrm{mM} \mathrm{MgCl} \mathrm{MgH}_{2} \mathrm{O}$, $10 \mathrm{mM}$ HEPES, and $10 \mathrm{mM}$ dextrose at $37^{\circ} \mathrm{C}$. The baseline internal diameter and changes in diameter in response to vasoactive substances were recorded at $80 \mathrm{~mm} \mathrm{Hg}$ and $37^{\circ} \mathrm{C}$ using MyoVIEW II 2.0 software (Danish Myo Technology, Aarhus, Denmark). All MAs were challenged with $3 \mu \mathrm{M}$ PE to test their reactivity before experiments were performed. MAs that did not contract upon PE addition were discarded. Data are expressed as the percentage of buffer baseline.

Wire Myography. The methods used were described previously (Russell and Watts, 2000) with modification. Aortas from RGS2 $2^{+/+}$ and RGS2 $2^{-1-}$ mice were isolated free of fat and connective tissue and cut into rings ( 2 to $3 \mathrm{~mm}$ in length). The aortic rings were mounted on two L-shaped stainless-steel wires inserted into the lumen and immersed in muscle baths filled with warm, aerated PSS containing $130 \mathrm{mM} \mathrm{NaCl}, 4.7 \mathrm{mM} \mathrm{KCl}, 1.18 \mathrm{mM} \mathrm{KH}_{2} \mathrm{PO}_{4}, 1.17 \mathrm{mM} \mathrm{MgSO}_{4} \cdot 7 \mathrm{H}_{2} \mathrm{O}$,
$1.6 \mathrm{mM} \mathrm{CaCl} 2 \cdot 2 \mathrm{H}_{2} \mathrm{O}, 14.9 \mathrm{mM} \mathrm{NaHCO}{ }_{3}, 5.5 \mathrm{mM}$ dextrose, and $0.03 \mathrm{mM} \mathrm{CaNa}{ }_{2} \mathrm{EDTA}$. One end of the preparation was attached to a stainless-steel rod, and the other end was attached to a force transducer (FT03; Grass Instruments, Quincy, MA). The basal tension of the aortic ring was maintained at $0.5 \mathrm{~g}$. Changes in isometric force after adding PE in a cumulative manner were recorded on a Grass polygraph (Grass Instruments).

Reversible Permeabilization. Reversible permeabilization of isolated MAs to introduce RGS2 plasmids to the MAs was achieved by a modification of previous methods (Lesh et al., 1995; Welsh et al., 2002). Briefly, MAs from RGS2 ${ }^{-/-}$mice were loaded with RGS2-GFP constructs by sequential incubation in the following solutions: 1) $10 \mathrm{mM}$ EGTA, $120 \mathrm{mM} \mathrm{KCl}, 5 \mathrm{mM} \mathrm{Na} \mathrm{A}_{2} \mathrm{ATP}, 2 \mathrm{mM}$ $\mathrm{MgCl}_{2}$, and $20 \mathrm{mM}$ 2-\{[2-hydroxy-1,1-bis(hydroxymethyl)ethyl] amino\}ethanesulfonic acid (TES) ( $\mathrm{pH} 6.8 ; 120$ minutes, $\left.4^{\circ} \mathrm{C}\right)$; and 2) $0.1 \mathrm{mM}$ EGTA, $120 \mathrm{mM} \mathrm{KCl}, 5 \mathrm{mM} \mathrm{Na} \mathrm{ATP}_{2} \mathrm{AT}, 2 \mathrm{mM} \mathrm{MgCl}_{2}, 20 \mathrm{mM}$ TES, and $1 \mu \mathrm{g} / \mathrm{ml}$ RGS2-GFP plasmids ( $\mathrm{pH} 6.8$; overnight, $4^{\circ} \mathrm{C}$ ). Subsequently, arteries were transferred to the following solutions: 3) $0.1 \mathrm{mM}$ EGTA, $120 \mathrm{mM} \mathrm{KCl}, 5 \mathrm{mM} \mathrm{Na}{ }_{2} \mathrm{ATP}, 10 \mathrm{mM} \mathrm{MgCl}_{2}$, and $20 \mathrm{mM}$ TES (pH 6.8; 30 minutes, $4^{\circ} \mathrm{C}$ ); and 4) $140 \mathrm{mM} \mathrm{NaCl}, 5 \mathrm{mM}$

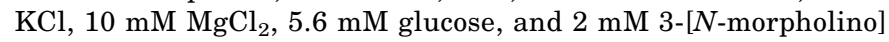

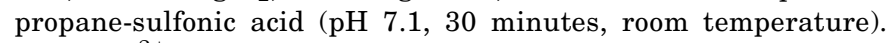
Then $\left[\mathrm{Ca}^{2+}\right]$ was gradually increased from 0.001 to 0.01 to 0.1 to 1.6-2.4 mM every 10 minutes by adding $\mathrm{CaCl}_{2}$ to solution 4 . Arteries were then incubated in Dulbecco's modified Eagle's medium/F-12 culture medium (catalog number 11039021; Gibco, Carlsbad, CA) supplemented with $50 \mathrm{U} / \mathrm{ml}$ penicillin and $50 \mathrm{mg} / \mathrm{ml}$ streptomycin and maintained at $37^{\circ} \mathrm{C}, 5 \% \mathrm{CO}_{2}$ in an incubator. MAs expressing RGS2-GFP were used for myography 24-48 hours after being transferred to culture medium.

Immunofluorescence. After reversible permeabilization, MAs were fixed in $4 \%$ paraformaldehyde for 24 hours and rinsed with phosphate buffer (PB), $\mathrm{pH}$ 7.2. The arteries were permeabilized in DMSO $100 \%$ for 10 minutes, then incubated in blocking buffer/PB buffer containing 4\% donkey serum (catalog number D9663, lot SLBQ9773V; Sigma-Aldrich) for 30 minutes, and then incubated with rabbit anti-GFP antibody (catalog number A6455; Life Technologies, Carlsbad, CA) at 1:500 dilution at $4^{\circ} \mathrm{C}$ overnight. The arteries were then rinsed with $0.2 \%$ Triton X-100 in PBS and incubated in Cy3/anti-rabbit secondary antibody (code 711-165-152, lot 131748; Jackson ImmunoResearch, West Grove, PA) at 1:300 dilution at room temperature. The arteries were mounted using ProLong Gold Antifade Mountant with 4',6-diamidino-2-phenylindole (catalog number P36941; Life Technologies) and visualized using a $40 \times$ objective in an Olympus FluoView 1000 laser scanning confocal microscope.

Cell Culture and Transfection. Cell lines were maintained in a humidified incubator at $37^{\circ} \mathrm{C}$ with $5 \% \mathrm{CO}_{2}$. Human embryonic kidney (HEK)-293 cells and Chinese hamster ovary (CHO)-K1 cells (ATCC, Manassas, VA) were maintained in Dulbecco's modified Eagle's medium (Gibco) and F-12 nutrient mixture (Gibco), respectively, supplemented with 10\% FBS, $100 \mathrm{U} / \mathrm{ml}$ penicillin, and $100 \mathrm{mg} / \mathrm{ml}$ streptomycin. X-tremeGENE HP DNA Transfection Reagent (Roche Life Sciences) and DNA-In CHO (MTI-GlobalStem, Gaithersburg, MD) were used to transfect HEK-293 cells and CHO-K1 cells according to the manufacturers' recommended protocols. Transfection mixes were prepared in antibiotic-free Opti-MEM (Gibco). Experiments were run 24-48 hours after transfection.

Confocal Microscopy. HEK-293 cells were seeded onto collagencoated 35-mm glass-bottom Petri dishes (MatTek Corporation, Ashland, MA) and transfected with RGS2-GFP plasmid constructs along with plasmid DNA encoding $\mathrm{G}_{\mathrm{q}}^{\mathrm{Q} 209 \mathrm{~L}}$ or pcDNA3.1 as the control. An Olympus FluoView 1000 laser scanning confocal microscope with a $60 \times$ oil immersion objective using 488-nm excitation and 505- to 530 $\mathrm{nm}$ emission wavelengths for GFP was used for live cell imaging. This experiment was done 24 hours post-transfection. Representative images of at least 80 live cells per condition from three independent 
experiments were acquired. The line scan analysis function in ImageJ (Schneider et al., 2012) was used to perform densitometric quantitation of protein expression.

$\mathbf{C a}^{2+}$ Mobilization Assay. CHO cells were seeded into black, flat, clear-bottom 384-well plates (Greiner Bio-one, Kremsmünster, Austria) 24 hours post-transfection. Cells were allowed to attach overnight. After the media were removed, cells were loaded with $1 \times$ Fluo- 4 NW (Molecular Probes, Eugene, OR) in a loading buffer containing Hanks' basal saline solution and $20 \mathrm{mM}$ HEPES, pH 7.4, supplemented with $2.5 \mu \mathrm{M}$ probenecid. The cell plates were incubated for 30 minutes at $37^{\circ} \mathrm{C}$, followed by 30 minutes at room temperature. The Fluo-4 NW was removed and loading buffer was added at $20 \mu \mathrm{l} /$ well to the cell plate. A range of concentrations of AngII (catalog number A9525; Sigma) at $2 \times$ the final concentration was freshly prepared in loading buffer supplemented with $0.1 \%$ bovine serum albumin in a ligand plate for automated injection into the wells at $20 \mu \mathrm{l} /$ well by a FDSS $/ \mu$ Cell kinetic fluorescence plate reader (Hamamatsu Photonics, Japan). Assay plates were placed in the plate reader and changes in intracellular free calcium in response to the receptor activation were measured.

Statistical Analyses. Data are presented as means \pm S.E. Vessel diameter data were analyzed by the $t$ test or two-way ANOVA followed by the indicated multiple-comparisons test. All statistical analyse were performed using GraphPad Prism 6.0 software (GraphPad, San Diego, CA). Statistical significance was denoted by $P<0.05$ or as indicated in the figure legends.

\section{Results}

RGS2 Deficiency Enhances Vascular Contractility to PE and AngII and Causes Impairment of ACh-Mediated Vasorelaxation. Vascular responses of RGS2-deficient arteries to vasoconstrictors and vasodilators have been extensively studied in different vascular beds, including the aorta, MA, renal interlobar, and uterine artery (Tang et al., 2003; Hercule et al., 2007; Osei-Owusu et al., 2012; Jie et al., 2016). Here we assessed the response of MA and aorta to PE, AngII, and ACh with the goals of: 1) validating the effect of RGS2 deficiency on vascular reactivity and 2) optimizing experimental conditions for studies of RGS2 mutant proteins in the context of vascular tissue. Second-order MAs and rings from the thoracic aorta from male $\mathrm{RGS}^{+/+}$and RGS2 $2^{-/-}$mice were used to determine how RGS2 protein affects vascular reactivity to different vasoactive substances. $\mathrm{PE}$, which activates the $\mathrm{G} \alpha \mathrm{q}$-coupled $\alpha 1$ adrenergic receptor, caused a concentration-dependent contraction in $\mathrm{RGS2}^{+/+}$MAs and aorta; this response was significantly augmented in $\mathrm{RGS}^{-1-}$ aortic rings in wire myography (Fig. 1B) but not in pressurized MAs from RGS2 $2^{-I-}$ mice (Fig. 1A). The MAs isolated from RGS2 $2^{-1-}$ mice did show increased responsiveness to $5 \mathrm{nM}$ AngII $(28 \% \pm 1.8 \%$ contraction in RGS2 $2^{-/-}$vs. $10 \% \pm 2.6 \%$ in RGS2 ${ }^{+/+}, P<0.01, t$ test; Fig. 1C; Supplemental Fig. 1A). In addition, the maximum of AChmediated vasodilation in RGS2 ${ }^{-1-}$ MA preconstricted with $3 \mu \mathrm{M}$ $\mathrm{PE}$ was markedly impaired $(48.6 \% \pm 8.4 \%$ relaxation in $\mathrm{RGS}^{-/-}$vs. $92.7 \% \pm 6.2 \%$ relaxation in $\mathrm{RGS2}^{+/+}, P<0.01$ and $P<0.0001$, respectively, in two-way ANOVA with the Sidak post-test; Fig. 1D). We chose to focus on testing the reactivity of MAs expressing RGS2 mutants to AngII and ACh in subsequent experiments because MAs contribute to vascular resistance, which determines arterial blood pressure (Christensen and Mulvany, 1993).

Expression of RGS2-GFP in Cultured RGS2 ${ }^{-1-}$ MAs. Using reversible permeabilization to deliver plasmids into intact arteries, we were able to express RGS2-GFP constructs in MAs. Immunofluorescent staining of GFP showed that RGS2-GFP was present in the MA between 48 and 72 hours after reversible permeabilization but absent with a pcDNA3.1 transfection control (Fig. 2A). The staining also showed that the Q2L-GFP appeared to have lower intensity compared with WT and other mutant RGS2 in the MAs, which aligned with the low protein level of the Q2L mutant in CHO cells (Phan et al., 2017). Further characterization was done by performing
A

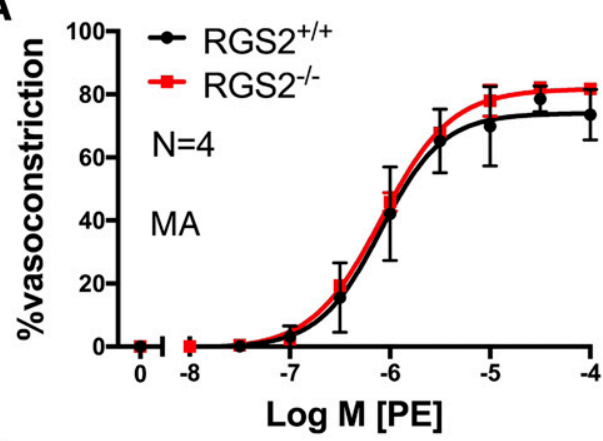

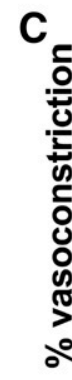

B

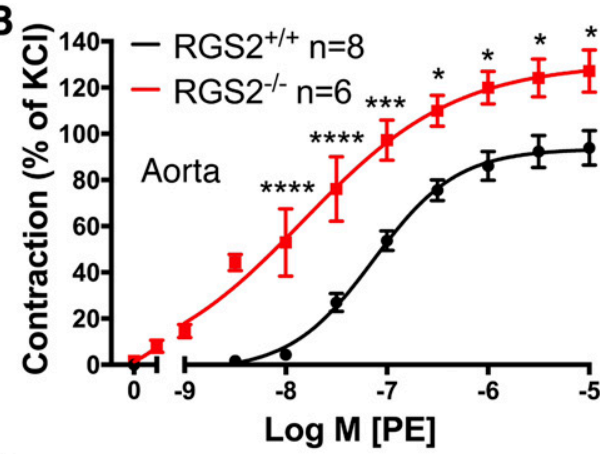

D

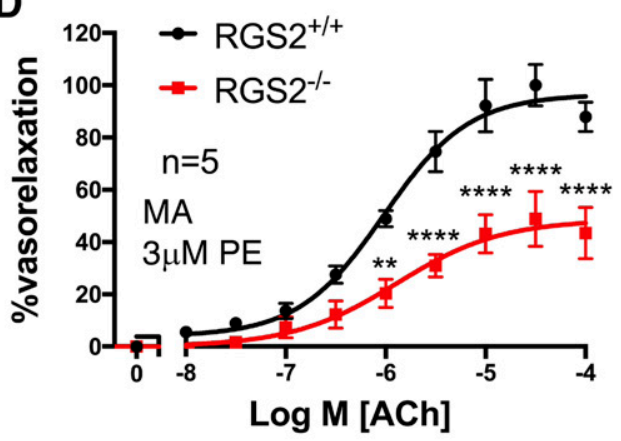

Fig. 1. Vascular reactivity of $\mathrm{RGS}^{+/+}$ and $\mathrm{RGS2}^{-1-}$ arteries to vasoconstrictor $\mathrm{PE}$ and AngII and vasodilator ACh. (A) MAs from RGS2 ${ }^{+/+}$and $\mathrm{RGS}^{-/-}$mice responded similarly to different concentrations of PE. (B) Aorta isolated from $\mathrm{RGS}^{-/-}$mice showed greater sensitivity to $\mathrm{PE}$ than aorta from RGS2 $2^{+/+}$mice. (C) MA response to AngII (5 nM). (D) Concentration response of ACh-induced vasodilation expressed as percentages of the increase in arterial diameter after constriction with PE $\left(3 \times 10^{-6} \mathrm{M}\right)$ $(n=5)$. Data are expressed as the mean \pm S.E. $* P<0.05 ; * * P<0.01$; $* * * P<0.001 ; * * * * P<0.0001$ vs. WT (two-way ANOVA with Sidak post-test). $n$ indicates the number of mice used to isolate vessels for each experiment. 


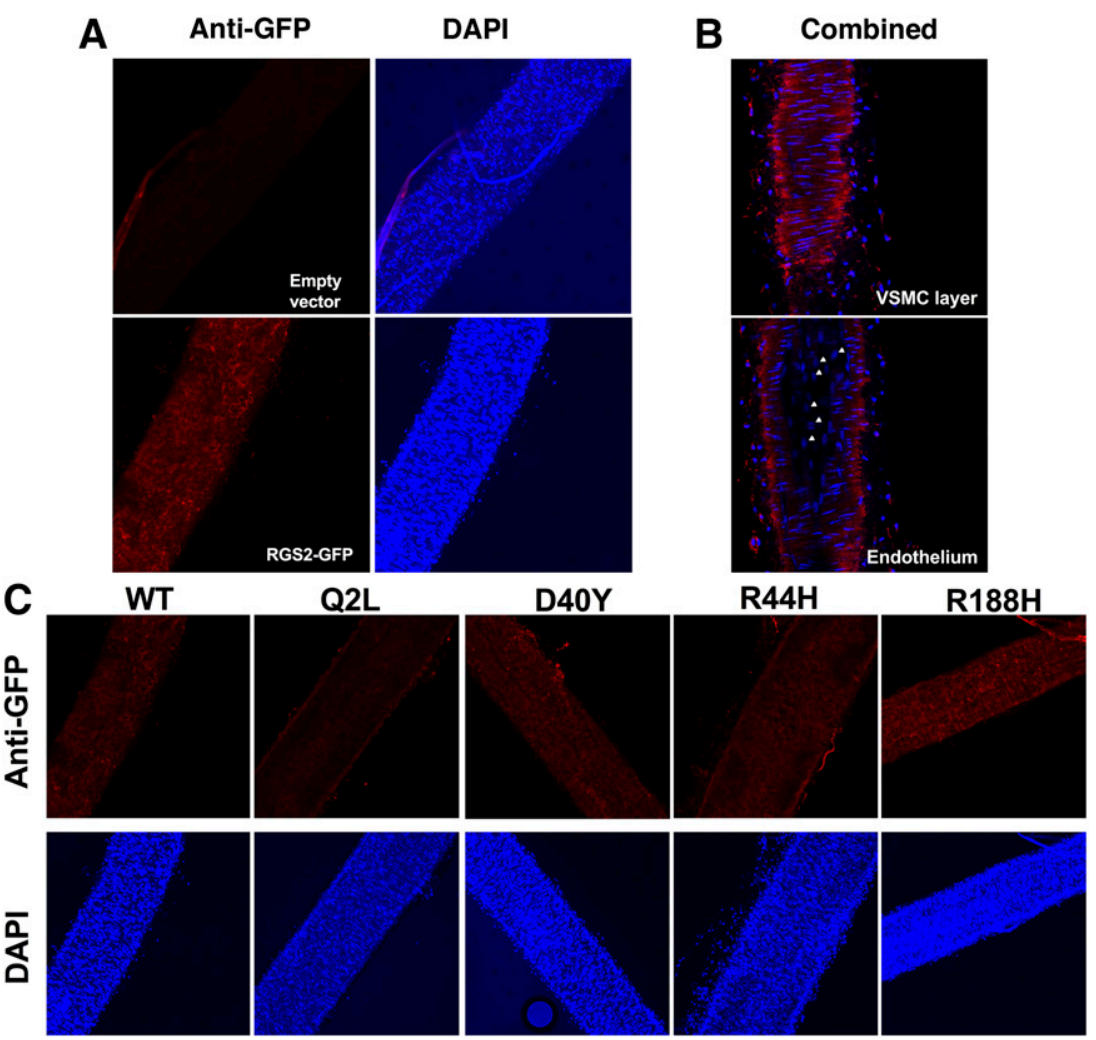

Fig. 2. Reversible permeabilization permits transfection of RGS2-GPF into RGS2 ${ }^{-/-}$MAs. Two to three days after reversible permeabilization (described in the Materials and Methods), arteries were stained for rabbit GFP and labeled with Cy3/anti-rabbit secondary antibody. The arteries were mounted to slides using ProLong Gold Antifade Mountant and visualized using confocal microscopy. (A) Expression of RGS2-GFP was detected compared with arteries exposed to empty vector. (B) RGS2-GFP was overexpressed in VSMCs but not endothelial cells (arrowheads). (C) Representative images showed whole vessel expression of RGS2GFP. The expression level of the Q2L mutant in MAs was lower than that of RGS2-WT and other mutant RGS2 proteins. DAPI, 4',6-diamidino-2-phenylindole. The round circle below the cell in the middle DAPI image is an artifact that appears to be a bubble.

z-stack imaging by confocal microscopy. RGS2-GFP was expressed in the VSMC layer and virtually absent RGS2GFP in the endothelial cell layer (Fig. 2B), based on the depth in the z-stack and morphology of cell nuclei/elongated nuclei in VSMCs and round nuclei in endothelial cells (Luo et al., 2016).

RGS2 LOF Mutations Fail to Suppress AngII-Induced Vasoconstriction. To examine the effect of RGS2 mutants in regulating vasoconstriction, we expressed plasmid constructs encoding RGS2 WT and mutants in RGS2 ${ }^{-/-}$MAs by reversible permeabilization versus empty vector as the control. For these experiments, we used a higher concentration of AngII for cultured MAs (50 nM instead of $5 \mathrm{nM}$ in freshly isolated arteries). In pilot studies with transfected RGS2 knockout MAs, $30 \mathrm{nM}$ AngII gave only a small contractile response, whereas $50 \mathrm{nM}$ gave a response similar to that elicited by 5 and $50 \mathrm{nM}$ in untransfected MAs (Supplemental Fig. 1B). The 50 $\mathrm{nM}$ AngII provided sufficient significant vasoconstriction to be able to observe effects of RGS2 in transfected arteries. This is potentially due to impaired receptor function after reversible permeabilization. MAs expressing empty vector or $\mathrm{RGS}^{-1-}$ MAs without RGS2 transfection showed $34.3 \% \pm 4.7 \%$ (Fig. 3A) and $30.0 \% \pm 3.1 \%$ (Fig. 3B) contraction relative to baseline, respectively. Expression of RGS2 WT was able to suppress contraction in two independent experiments. In MAs expressing RGS2 WT, AngII caused a reduction in arterial diameter of only $4.2 \% \pm 1.3 \%(P<0.01$, one-way ANOVA, Dunnett post-test; Fig. 3A) and $7.2 \% \pm 2.6 \%(P<0.05$, oneway ANOVA, Dunnett post-test; Fig. 3B). Two mutants identified in our previous work as having normal function, RGS2 M5V and A99G (Phan et al., 2017), resulted in a similar level of vascular contraction to RGS2 WT $(6.8 \% \pm 3.6 \%$ and $10.9 \% \pm 4.2 \%$, respectively; Fig. $3 \mathrm{~B})$. The RGS2 Q2L mutant gave reduced suppression of contraction compared with RGS WT $(18.8 \% \pm 6.6 \%$ constriction vs. $4.2 \% \pm 1.3 \%)$; however, the difference was not statistically significant $(P=0.09)$ (Fig. 3A). Arteries expressing RGS2 D40Y, $\mathrm{R} 44 \mathrm{H}$, and $\mathrm{R} 188 \mathrm{H}$ mutants showed a significantly elevated contractile response to $50 \mathrm{nM}$ AngII stimulation $(28.3 \% \pm$ $7.7 \%, 25.7 \% \pm 8.7 \%$, and $29.8 \% \pm 5.5 \%$, respectively) compared with arteries expressing RGS2 WT $(4.2 \% \pm$ $1.3 \%, P<0.05$, one-way ANOVA, Dunnett post-test) (Fig. 3).

RGS2 LOF Mutations Differentially Affect ACh-Evoked Vasodilation. Because freshly isolated $\mathrm{RGS}^{-1-}$ MAs show reduced relaxation to $\mathrm{ACh}$, LOF mutations of RGS2 may behave similarly. To probe the function of these mutants in mediating $\mathrm{ACh}$-induced vascular relaxation, we measured ACh-evoked vasodilation of MAs expressing WT and mutant RGS2. Using MAs preconstricted with PE $(3 \mu \mathrm{M})$, we found that cultured RGS2-deficient MA (transfected with empty vector) showed a maximal relaxation of $52.8 \% \pm 14.3 \%$ (Fig. 4). This is similar to the response of freshly isolated $\mathrm{RGS2}^{-1-}$ MAs $(48.6 \% \pm 8.4 \%$; Fig. 1C) and much less than that of arteries expressing WT RGS2 $(99.6 \% \pm 5.5 \%, P=0.0001$, two-way ANOVA with Dunnett post-test; Fig. 4). Among the four LOF mutants, we found that ACh-mediated relaxation with the RGS2 Q2L and R188H mutants was significantly impaired compared with RGS2 WT (Q2L: 75\% $\pm 7 \%, P<0.05$; R188H: $63.8 \% \pm 14.3 \%, P<0.001$, Dunnett post-test; Fig. 4A). Arteries expressing the D40Y and R44H mutant RGS2, however, showed an equivalent relaxation response to those with RGS2 WT (D40Y: 98.4\% $\pm 12.3 \%$; R44H: $93.98 \% \pm$ $4.8 \%$; Figs. $4 \mathrm{~B}$ and $5 \mathrm{E}$ ). It is notable that these latter two mutations reduce RGS2 function by perturbing membrane localization, whereas the other two have different mechanisms (Phan et al., 2017). 

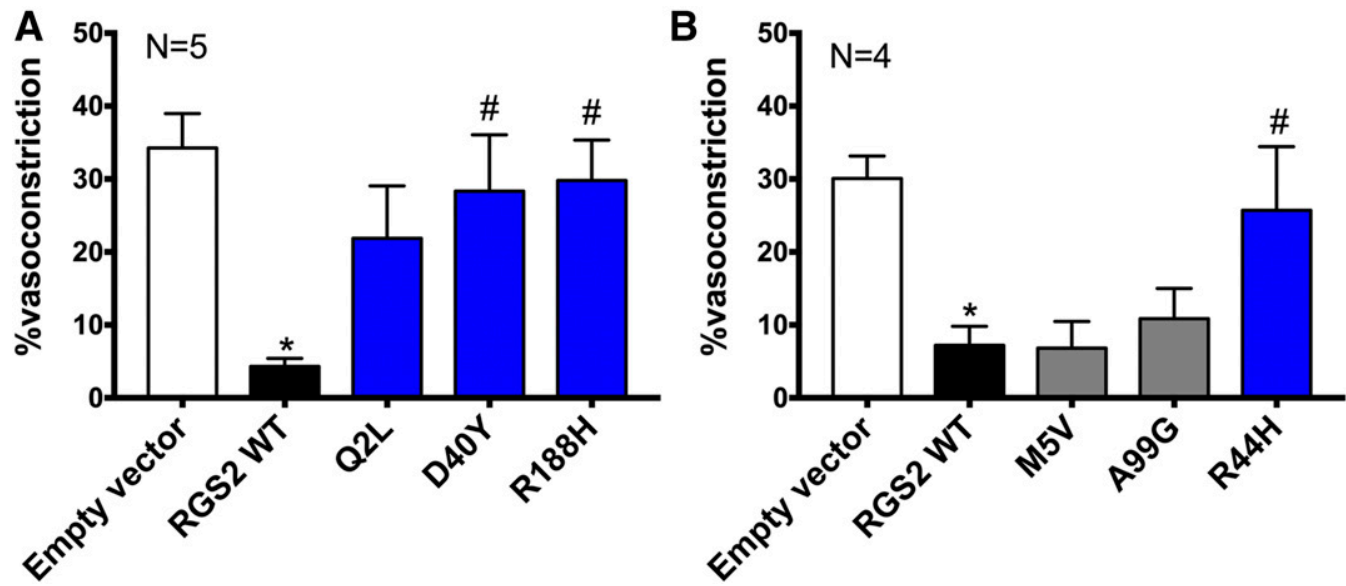

Fig. 3. LOF mutations of RGS2 failed to suppress AngII-mediated vasoconstriction. RGS2-GFP constructs were introduced to MAs isolated from $\mathrm{RGS}^{-1-}$ mice by reversible permeabilization. (A) RGS2 WT- and M5V- and A99G-expressing (two normal function mutations; Phan et al., 2017) arteries reduced vascular contraction upon $50 \mathrm{nM}$ AngII stimulation, whereas the other mutants did not. (B) D40Y-, R44H-, and R188H mutant-expressing arteries showed a significantly elevated contractile response to $50 \mathrm{nM}$ AngII stimulation compared with RGS2 WT-expressing arteries. ${ }^{*} P<0.05$ compared with empty vector; ${ }^{\#} P<0.05$ compared with WT (one-way ANOVA with Dunnett post-test).

Ser46 and Ser64 Phosphomimetic Mutants of D40Y and R44H RGS2 can Rescue Protein Plasma Membrane Localization and Function. In VSMCs, RGS2 has been shown to be an effector of the NO-cGMP pathway. RGS2 is phosphorylated by protein kinase G $1 \alpha$ (PKG1 $\alpha)$, which is activated by cGMP; as a consequence, RGS2 membrane localization and GTPase accelerating protein (GAP) function is enhanced (Sun et al., 2005; Osei-Owusu et al., 2007). RGS2 phosphorylation at Ser46 and Ser64 by PKG1 $\alpha$ increased plasma membrane (PM) localization and GAP activity of RGS2 protein (Tang et al., 2003; Osei-Owusu et al., 2007). Given that the two RGS2 mutants mentioned above (D40Y, $\mathrm{R} 44 \mathrm{H})$ had impaired PM localization in HEK-293 cells but supported a normal ACh-evoked relaxation response in the MAs, we hypothesized that phosphorylation of D40Y and R44H mutant proteins in VSMCs by NO-cGMP-PKG pathway activation upon $\mathrm{ACh}$ stimulation is able to rescue the impaired membrane localization and function of these mutants. The effects of site-specific phosphorylation on protein activity can be mimicked by mutation of Ser (S) or Thr (T) to Asp (D).
Therefore, to model the effects of phosphorylation of Ser46 and Ser64 on RGS2 protein localization, we performed mutagenesis to make S46D and S64D mutations of tagged constructs encoding WT, D40Y, and R44H (RGS2 WT DD, D40Y DD, and $\mathrm{R} 44 \mathrm{H} \mathrm{DD})$. The expression levels of the phosphomimetic mutants were similar to their nonphosphorylated proteins and similar to WT RGS2 (Supplemental Fig. 2). When coexpressed with G?q Q209L, D40Y, and R44H, mutant RGS2 remained in the nucleus, whereas RGS2 WT and the phosphomimetic mutants (RGS2 DD, D40Y DD, and R44H DD) localized to the PM (Fig. 5, A and B). In addition, the RGS2 $\mathrm{D} 40 \mathrm{Y}$ and $\mathrm{R} 44 \mathrm{H}$ mutants supported less $\mathrm{Ca}^{2+}$ inhibition compared with RGS2 WT (Fig. 5C), whereas RGS2 D40Y DD and R44H DD suppressed AngII receptor type 1-mediated $\mathrm{Ca}^{2+}$ mobilization equivalently to RGS2 DD (Fig. 5D).

Effect of PKG Inhibition on ACh-Mediated Relaxation in MAs Expressing the D40Y RGS2 Mutant. If phosphorylation helps maintain the function of the D40Y mutant RGS2, then inhibition of PKG with Rp-8-pCPTcGMPS $(30 \mu \mathrm{M})$ should prevent the rescue of relaxation
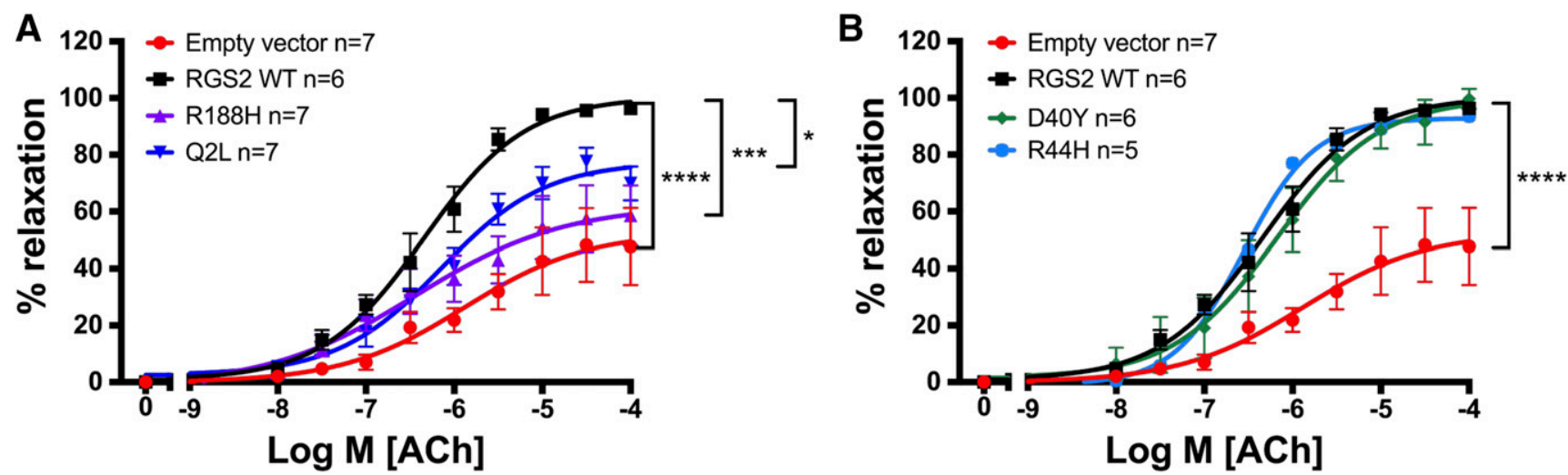

Fig. 4. Differential effects of RGS2 missense mutations on ACh-mediated vasorelaxation. (A) RGS2-GFP expression in RGS2 ${ }^{-1-}$ MAs rescued AChmediated relaxation. RGS2 WT-expressing arteries showed enhanced relaxation compared with empty vector transfected arteries. Q2L- and R188Hexpressing arteries showed less relaxation compared with RGS2 WT-expressing arteries. (B) D40Y- and R44H-expressing arteries, however, showed a comparable relaxation response to RGS2 WT. Data are expressed as the mean \pm S.E. $* P<0.05 ; * * * P<0.001 ; * * * * P<0.0001$ vs. WT (two-way ANOVA with Dunnett post-test). 
A

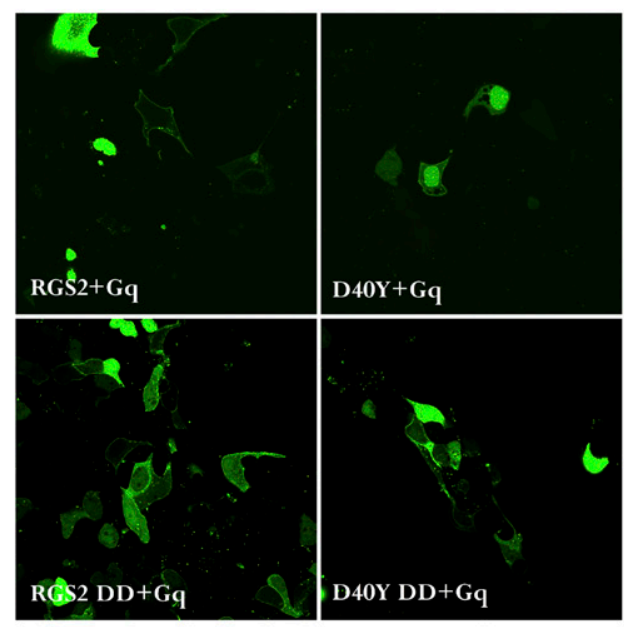

C

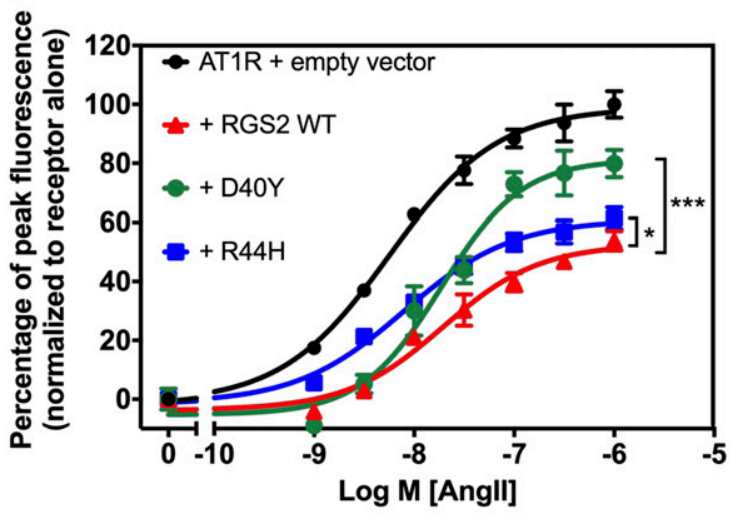

E

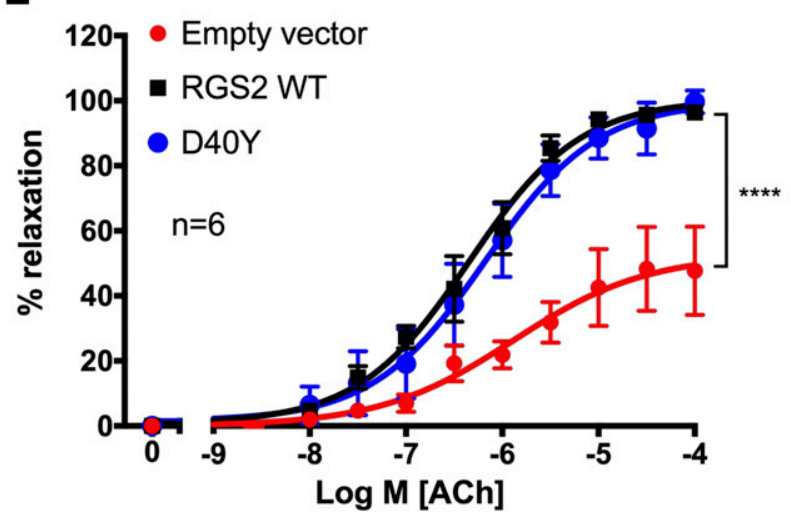

B

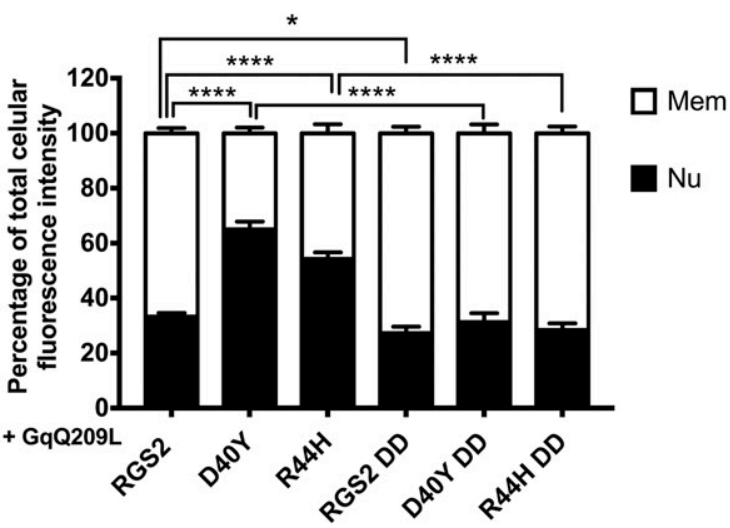

Fig. 5. Effects of phosphorylation on RGS2 localization and function. (A) Representative confocal images showing that phosphomimetic mutations of D40Y and R44H rescued the mutants' membrane localization and function in HEK-293 cells. When coexpressed with G?q, Q209L, D40Y, and R44H remained in the nucleus, whereas RGS2 WT, RGS2 DD, D40Y DD, and R44H DD localized to the PM. (B) Quantification of protein localization of at least 80 live cells from three to four independent experiments. ${ }^{*} P<0.05 ; * * * P<0.0001$ (using one-way ANOVA with Bonferroni post-test). (C) LOF phenotype of the D40Y and R44H mutant RGS2 in $\mathrm{Ca}^{2+}$ assay in CHO cells. (D) D40Y DD and R44H DD suppressed AT1R-mediated Ca ${ }^{2+}$ mobilization as well as RGS2 DD. (E) The D40Y mutant RGS2 supported a normal ACh-mediated relaxation response. (F) PKG inhibition by $30 \mu$ M Rp-8-pCPT-cGMPS significantly reduced vascular relaxation in D40Y-expressing MAs. $n$ indicates the number of mice used to isolate vessels for each experiment. Data are expressed as the mean \pm S.E. $* P<0.05$; ${ }^{* * *} P<0.001$; $* * * * P<0.0001$ (using two-way ANOVA with Dunnett post-test). AT1R, AngII receptor type $1 ;$ n.s., not significant.

responses of MAs. PKG inhibition slightly reduced maximal relaxation of MAs expressing WT RGS2 from $99.6 \% \pm 5.5 \%$ to $87.8 \% \pm 4.2 \%$ (not significant) and shifted the ACh concentration-response curve a half-log to the right $\left(\operatorname{logEC} \mathrm{C}_{50}\right.$ $-5.8 \pm 0.06$ vs. $-6.3 \pm 0.09$, not significant; Fig. $5, \mathrm{E}$ and F; Table 1). The relaxation response of RGS2 ${ }^{-l-}$ MAs transfected with empty vector was impaired (maximum relaxation $52.9 \% \pm 14.3 \%$ ) and was further depressed by PKG inhibition (maximum relaxation $38.4 \% \pm 3.5 \%$, not significant; Fig. 5, E and F; Table 1). However, PKG inhibition drastically reduced the dilation of MAs transfected with the D40Y mutant RGS2 $(40.3 \% \pm 6.7 \%$ vs. $98.4 \% \pm 12.3 \%, P<0.001$; Fig. 5 , E and F; 
TABLE 1

Relaxation of MAs expressing RGS2 WT and D40Y to ACh demonstrated by maximum and $\mathrm{EC}_{50}$ values derived from $\mathrm{ACh}$

concentration-relaxation response curves

Data are presented as the mean \pm S.E.M.

\begin{tabular}{lllc}
\hline RGS2 Mutant/Treatment & $n$ & \multicolumn{1}{c}{ Maximum } & $\operatorname{LogEC}_{50}$ \\
\hline Empty vector & 7 & $52.9 \pm 14.3$ & $-5.9 \pm 0.43$ \\
RGS2 WT & 6 & $99.6 \pm 5.5^{* * *}$ & $-6.3 \pm 0.09$ \\
D40Y & 6 & $98.4 \pm 12.3^{*}$ & $-6.2 \pm 0.19$ \\
Empty vector + PKG inhibitor & 4 & $38.4 \pm 3.5^{\# \#}$ & $-6.3 \pm 0.14$ \\
RGS2 WT + PKG inhibitor & 4 & $87.8 \pm 4.2$ & $-5.8 \pm 0.06$ \\
D40Y + PKG inhibitor & 4 & $40.3 \pm 6.7^{\# \#, ~ \& \& \&}$ & $-6.2 \pm 0.22$
\end{tabular}

${ }^{*} P<0.05$ compared with empty vector; ${ }^{* *} P<0.01$; ${ }^{\# \#} P<0.01$ compared with RGS2 WT + PKG inhibitor; ${ }^{\& \&} P<0.001$ compared with D40Y.

Table 1). The marked reduction of relaxation response in MAs expressing the D40Y mutant is consistent with a model in which lack of phosphorylation and PM localization occurs when PKG1 $\alpha$ is inhibited. We did not test MAs expressing the $\mathrm{R} 44 \mathrm{H}$ mutant in this experimental setting, but given the similarity in mechanisms of the D40Y and $\mathrm{R} 44 \mathrm{H}$ at the molecular level, we think the MAs expressing $\mathrm{R} 44 \mathrm{H}$ might behave similarly to the MAs expressing D40Y.

\section{Discussion}

In this study, we examined the physiologic effects of human RGS2 LOF mutations in a resistance artery. Four mutant RGS2 proteins (Q2L, D40Y, R44H, R188H) were previously identified as LOF mutations using a $\mathrm{Ca}^{2+}$ mobilization phenotypic assay in CHO cells (Phan et al., 2017). Here, we report that all four mutant proteins failed to effectively suppress AngII-mediated vasoconstriction in mouse MAs. Interestingly, only the Q2L and R188H mutations exhibited impaired ACh-mediated relaxation. The $\mathrm{D} 40 \mathrm{Y}$ and $\mathrm{R} 44 \mathrm{H}$ RGS2 mutants supported a normal relaxation response to $\mathrm{ACh}$. We also provide mechanistic evidence that the differential relaxation supported by mutant RGS2 proteins may be attributed to phosphorylation of RGS2 by PKG in VSMCs. It appears that PKG-dependent phosphorylation can rescue PM localization in HEK-293 cells and restore the vasorelaxation function of the D40Y and probably the R44H mutant RGS2 in MAs.

Freshly Isolated MA Responses to AngII and ACh. We were able to confirm the previously published effects of ACh in WT and RGS2-deficient MAs (Osei-Owusu et al., 2012). In addition, we show for the first time that MAs from $\mathrm{RGS}^{-1-}$ mice exhibit augmented vasoconstriction to AngII. This is consistent with previous literature showing that RGS2deficient renal interlobar arterioles had an increased contraction in response to AngII (Hercule et al., 2007).

ACh-Induced Relaxation of MAs Expressing RGS2 Mutant Proteins. One component of RGS2-mediated relaxation of VSMC involves PKG-mediated phosphorylation and membrane translocation of RGS2 to inhibit contraction evoked by Gq/11-coupled vasoconstrictor receptors (Tang et al., 2003; Sun et al., 2005). RGS2 also promotes endothelium-derived hyperpolarizing factor (EDHF)-dependent vasodilation by blunting Gi/o-dependent processes (Osei-Owusu et al., 2012). In our experiments, the RGS2-GFP proteins were expressed in the VSMC layer but did not appear to be expressed at significant levels in the endothelial cells (Fig. 2B). Therefore, we are not able to assess the EDHF-dependent relaxation effect of RGS2
(Osei-Owusu et al., 2012) with our method. However, overexpression of RGS2 in VSMC appears to be important in mediating ACh-evoked relaxation, since overexpression of the WT RGS2 in VSMCs was able to rescue the impaired $\mathrm{ACh}$-mediated relaxation in $\mathrm{RGS}^{-/-}$arteries. In addition, mutants with different LOF mechanisms (Phan et al., 2017) behaved differently (Fig. 4).

ACh mediates relaxation in part by activating the NOcGMP-PKG pathway in endothelium-intact arteries (Christopoulos and El-Fakahany, 1999; Sun et al., 2005). RGS2, as an effector of PKG, has previously been shown to be phosphorylated at Ser46 and Ser64 (Tang et al., 2003), enhancing PM localization. These phosphosites, interestingly, are located close to the sites of the D40Y and R44H mutations in RGS2, as shown in a model of the RGS2 N-terminal helix (Fig. 6) interacting with lipids (Tikhonova et al., 2006). This helix facilitates PM targeting (Heximer et al., 2001; Gu et al., 2007). Introducing phosphomimetic mutants at two PKG1 $\alpha$ phosphorylation sites of RGS2 (D40Y DD, R44H DD) in the D40Y and R44H mutant RGS2 proteins enhanced PM localization and function in suppressing $\mathrm{Ca}^{2+}$ release (Fig. $5, \mathrm{~A}, \mathrm{~B}$, and D). Conversely, PKG inhibition substantially reduced ACh-induced relaxation in RGS2 D40Y expressing RGS2 ${ }^{-/-}$ MAs (Fig. 5F). These results support our hypothesis that the normal ACh-mediated relaxation response of the D40Y and $\mathrm{R} 44 \mathrm{H}$ mutant RGS2 in MAs is due to phosphorylation of mutant proteins by PKG. At the molecular level, this phosphorylation may rescue PM localization of the mutant proteins. The RGS2 Q2L and R188H mutants may not be rescued by phosphorylation since the mechanism of their LOF behavior is not related to PM localization.

Although the RGS2 D40Y and R44H mutant alleles supported a normal relaxation response in resistance arteries, an intact endothelial NO-cGMP pathway was required for this response. When endothelial function is compromised such as in hypertension (Brandes, 2014) or in conditions wherein PKG function is deficient (Michael et al., 2008), RGS2 proteins expressed from these mutant alleles will likely be unphosphorylated, triggering aberrant $\mathrm{G} \alpha \mathrm{q}$ signaling and an impaired relaxation response.

How Does Phosphorylation of RGS2 at Ser46 and Ser64 Rescue D40Y and R44H Membrane Localization? The RGS2-lipid bilayer interaction was impaired in the R44H mutant according to the snorkeling-dependent stabilization model (Gu et al., 2008). In the case of the D40Y mutant, the change from a negatively charged Asp residue to a hydrophobic Tyr residue may disrupt the local environment for the anchorage of the adjacent Trp41 to the PM (Gu et al., 2008). Several mechanisms have been proposed to explain how phosphorylation at these sites might enhance recruitment of RGS2 to the PM. Salt bridge formation between the phosphorylated Ser residues and adjacent Lys residues (OseiOwusu et al., 2007) may stabilize the N-terminal amphipathic helix. This could explain how introduction of the Asp phosphomimetic in place of Ser at positions 46 and 64 could stabilize the amphipathic $\alpha$ helix disrupted by the D40Y and $\mathrm{R} 44 \mathrm{H}$ mutations to rescue function.

RGS2 plays three roles in the vasculature: 1) it functions directly as a GAP to negate vasoconstrictor actions through $\mathrm{G} \alpha \mathrm{q}, 2)$ it acts as an effector of NO-cGMP-PKG signaling to promote vasodilation by modulating $\mathrm{G} \alpha \mathrm{q}$-mediated vasoconstrictor action, and 3) it promotes EDHF-mediated vasorelaxation 


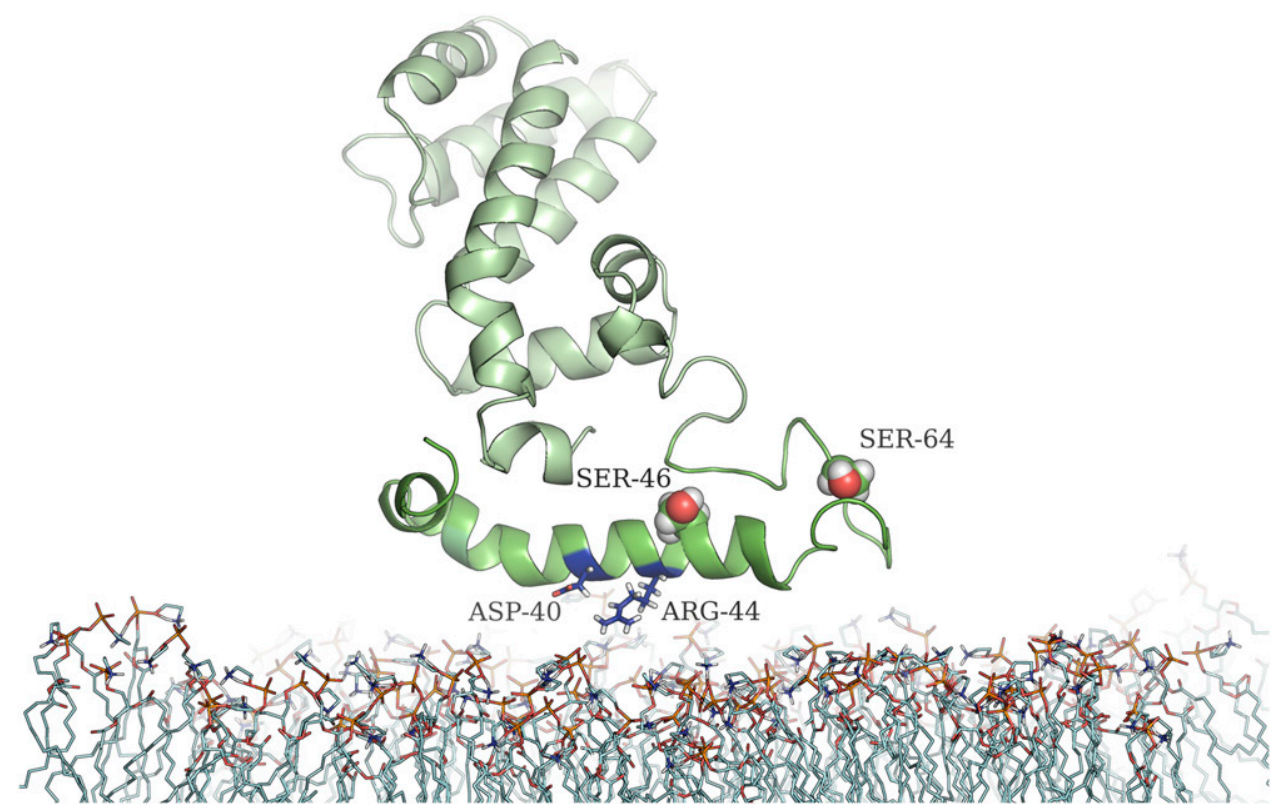

Fig. 6. Modeled structure of full-length RGS2 with lipid membrane. The N-terminal amphipathic $\alpha$-helix is orientated parallel with membrane, allowing interactions between amino acid side chains and components of lipid (Tikhonova et al., 2006). Mutated sites (D40, R44) are labeled in blue. Phosphorylated sites (S46, S64) are marked as spheres. The phosphate groups of lipids are in orange; the lipid acyl chains are in cyan.

(Osei-Owusu et al., 2012). By overexpressing RGS2 in the MAs, we were able to probe the first two functions of these RGS2 mutants in regulating vascular reactivity of MAs. A limitation of this study is the lack of knock-in animal models to allow investigation of whether endogenous RGS2 LOF mutants affect whole-body blood pressure responses. In addition, we are unable to assess their effects on the central nervous system or the kidney. Nevertheless, our data provide a new understanding how human RGS2 mutants behave in VSMCs in the resistance vasculature. We also provide molecular evidence for functional differences among mutant alleles where the function of $\mathrm{D} 40 \mathrm{Y}$ or $\mathrm{R} 44 \mathrm{H}$ mutants can be rescued by PKG-mediated phosphorylation. These results provide critical insights for precision therapeutic approaches to individuals bearing particular RGS2 mutations and may facilitate drug discovery to target these rare individuals.

\section{Acknowledgments}

The authors thank Jeffrey Leipprandt for mouse genotyping.

\section{Authorship Contributions}

Participated in research design: Phan, Neubig.

Conducted experiments: Phan.

Contributed new reagents or analytic tools: Jackson, Watts.

Performed data analysis: Phan, Neubig.

Wrote or contributed to the writing of the manuscript: Phan, Jackson, Shaw, Watts, Neubig.

Note Added in Proof-Financial support was accidentally not included in the Fast Forward version published on October 23, 2019. The funding footnote has now been added.

\section{References}

Bodenstein J, Sunahara RK, and Neubig RR (2007) N-terminal residues control proteasomal degradation of RGS2, RGS4, and RGS5 in human embryonic kidney 293 cells. Mol Pharmacol 71:1040-1050.

Brandes RP (2014) Endothelial dysfunction and hypertension. Hypertension 64: 924-928.

Christensen KL and Mulvany MJ (1993) Mesenteric arcade arteries contribute substantially to vascular resistance in conscious rats. J Vasc Res 30:73-79.

Christopoulos A and El-Fakahany EE (1999) The generation of nitric oxide by G protein-coupled receptors. Life Sci 64:1-15.

Diaz-Otero JM, Garver H, Fink GD, Jackson WF, and Dorrance AM (2016) Aging is associated with changes to the biomechanical properties of the posterior cerebral artery and parenchymal arterioles. Am J Physiol Heart Circ Physiol 310: H365-H375.

Gu S, He J, Ho WT, Ramineni S, Thal DM, Natesh R, Tesmer JJ, Hepler JR, and Heximer SP (2007) Unique hydrophobic extension of the RGS2 amphipathic helix domain imparts increased plasma membrane binding and function relative to other RGS R4/B subfamily members. J Biol Chem 282:33064-33075.

Gu S, Tirgari S, and Heximer SP (2008) The RGS2 gene product from a candidate hypertension allele shows decreased plasma membrane association and inhibition of Gq. Mol Pharmacol 73:1037-1043.

Hercule HC, Tank J, Plehm R, Wellner M, da Costa Goncalves AC, Gollasch M, Diedrich A, Jordan J, Luft FC, and Gross V (2007) Regulator of G protein signalling 2 ameliorates angiotensin II-induced hypertension in mice. Exp Physiol 92: $1014-1022$

Heximer SP, Knutsen RH, Sun X, Kaltenbronn KM, Rhee M-H, Peng N, Oliveira-dosSantos A, Penninger JM, Muslin AJ, Steinberg TH, et al. (2003) Hypertension and prolonged vasoconstrictor signaling in RGS2-deficient mice. $J$ Clin Invest 111: $445-452$.

Heximer SP, Lim H, Bernard JL, and Blumer KJ (2001) Mechanisms governing subcellular localization and function of human RGS2. $J$ Biol Chem 276: 14195-14203.

Jie L, Owens EA, Plante LA, Fang Z, Rensing DT, Moeller KD, and Osei-Owusu P (2016) RGS2 squelches vascular Gi/o and Gq signaling to modulate myogenic tone and promote uterine blood flow. Physiol Rep 4:e12692.

Lek M, Karczewski KJ, Minikel EV, Samocha KE, Banks E, Fennell T, O'DonnellLuria AH, Ware JS, Hill AJ, Cummings BB, et al.; Exome Aggregation Consortium (2016) Analysis of protein-coding genetic variation in 60,706 humans. Nature 536: $285-291$.

Lesh RE, Somlyo AP, Owens GK, and Somlyo AV (1995) Reversible permeabilization A novel technique for the intracellular introduction of antisense oligodeoxynucleotides into intact smooth muscle. Circ Res 77:220-230.

Luo T, Chen H, and Kassab GS (2016) 3D reconstruction of coronary artery vascular smooth muscle cells. PLoS One 11:e0147272.

Michael SK, Surks HK, Wang Y, Zhu Y, Blanton R, Jamnongjit M, Aronovitz M, Baur W, Ohtani K, Wilkerson MK, et al. (2008) High blood pressure arising from a defect in vascular function. Proc Natl Acad Sci USA 105:6702-6707.

Osei-Owusu P, Owens EA, Jie L, Reis JS, Forrester SJ, Kawai T, Eguchi S, Singh H, and Blumer KJ (2015) Regulation of renal hemodynamics and function by RGS2. PLoS One 10:e0132594.

Osei-Owusu P, Sabharwal R, Kaltenbronn KM, Rhee MH, Chapleau MW, Dietrich HH, and Blumer KJ (2012) Regulator of G protein signaling 2 deficiency causes endothelial dysfunction and impaired endothelium-derived hyperpolarizing factor-mediated relaxation by dysregulating Gi/o signaling. $J$ Biol Chem 287:12541-12549.

Osei-Owusu P, Sun X, Drenan RM, Steinberg TH, and Blumer KJ (2007) Regulation of RGS2 and second messenger signaling in vascular smooth muscle cells by cGMPdependent protein kinase. J Biol Chem 282:31656-31665.

Phan HTN, Sjögren B, and Neubig RR (2017) Human missense mutations in regulator of $\mathrm{G}$ protein signaling 2 affect the protein function through multiple mechanisms. Mol Pharmacol 92:451-458.

Russell A and Watts S (2000) Vascular reactivity of isolated thoracic aorta of the C57BL/6J mouse. J Pharmacol Exp Ther 294:598-604.

Schneider CA, Rasband WS, and Eliceiri KW (2012) NIH Image to ImageJ: 25 years of image analysis. Nat Methods 9:671-675.

Semplicini A, Lenzini L, Sartori M, Papparella I, Calò LA, Pagnin E, Strapazzon G, Benna C, Costa R, Avogaro A, et al. (2006) Reduced expression of regulator of G-protein signaling 2 (RGS2) in hypertensive patients increases calcium mobilization and ERK1/2 phosphorylation induced by angiotensin II. J Hypertens 24: 1115-1124. 
Sun X, Kaltenbronn KM, Steinberg TH, and Blumer KJ (2005) RGS2 is a mediator of nitric oxide action on blood pressure and vasoconstrictor signaling. Mol Pharmacol 67:631-639. Tang KM, Wang GR, Lu P, Karas RH, Aronovitz M, Heximer SP, Kaltenbronn KM Blumer KJ, Siderovski DP, Zhu Y, et al. (2003) Regulator of G-protein signaling-2 mediates vascular smooth muscle relaxation and blood pressure [published correction appears in Nat Med (2004) 10:105]. Nat Med 9:1506-1512.

Tikhonova IG, Boulègue C, Langer I, and Fourmy D (2006) Modeled structure of the whole regulator G-protein signaling-2. Biochem Biophys Res Commun 341:715-720.

Welsh DG, Morielli AD, Nelson MT, and Brayden JE (2002) Transient receptor potential channels regulate myogenic tone of resistance arteries. Circ Res 90:248-250.
Yang J, Kamide K, Kokubo Y, Takiuchi S, Tanaka C, Banno M, Miwa Y, Yoshii M, Horio T, Okayama A, et al. (2005) Genetic variations of regulator of G-protein signaling 2 in hypertensive patients and in the general population. J Hypertens 23: $1497-1505$

Address correspondence to: Richard R. Neubig, Department of Pharmacology and Toxicology, Michigan State University, B423 Life Sciences Bldg., 1355 Bogue St., East Lansing, MI 48824. E-mail: rneubig@msu.edu 